\title{
Cosmic ray measurements around the knee
}

\author{
Andrea Chiavassa ${ }^{\mathrm{a}}$ \\ Dipartimento di Fisica, Università degli Studi di Torino, via P. Giuria 1, 10125 Torino, Italy
}

\begin{abstract}
Primary cosmic rays of energy greater than $\sim 10^{14} \mathrm{eV}$ must be studied by indirect experiments measuring the particles generated in the EAS (Extensive Air Shower) development in atmosphere. These experiments are mainly limited by the systematic errors due to their energy calibration. I will discuss the main sources of these errors: the choice of the hadronic interaction model and of the mass of the primary particle (that cannot be measured on a event by event basis).

I will then summarize some recent measurements of the all particle spectrum, and I will show that, keeping into account the differences due to the energy calibration, they all agree on the spectral shape. Then I will describe the measurements of the light and heavy primaries mass groups spectra, discussing the claimed features. Using a simple calculation of the elemental spectra (based on the hypothesis that the knee energies follow a Peter's cycle) I will try to discuss if all these results can be interpreted in a common picture.
\end{abstract}

\section{Introduction}

Primary cosmic rays with energy greater than $10^{14} \mathrm{eV}$ cannot be studied by direct experiments operating on balloon or on satellites and their detection is only possible by means of indirect EAS experiments. All the main characteristics of the primary particle (i.e. mass, energy and arrival direction) must therefore be derived measuring the Extensive Air Showers (EAS) generated by the interaction of primary cosmic rays with atmospheric nuclei.

The experiments operating in the $10^{14}-10^{18} \mathrm{eV}$ energy range can be divided in two groups:

- Surface arrays: sampling the EAS at fixed atmospheric depth. Almost all of these arrays are able to simultaneously detect more than one EAS components: usually the electromagnetic and the muonic ones. Detecting the particle density and arrival times at different distances from the shower core these arrays derive the arrival direction of the primary cosmic ray, the number of charged particles $\left(N_{c h}\right)$ and the number of muons $\left(N_{\mu}\right)$ in the EAS at observation level. Both $N_{c h}$ and $N_{\mu}$ are derived as normalization of the lateral distribution of the particle density and can be defined either as the total number of particles at observation level or as the number of particles at a fixed distance from the shower core (distance that has to be fixed by every single experiment and depends both on the detector layout and on the primary energy range studied by the array). These detectors operate with a $100 \%$ duty cycle.

- Cherenkov arrays: detecting the cherenkov light emitted by particles during EAS development. The big advantage of these arrays is that they perform an almost

\footnotetext{
a e-mail: achiavas@to.infn.it
}

(C) The Authors, published by EDP Sciences. This is an Open Access article distributed under the terms of the Creative Commons Attribution License 4.0 (http://creativecommons.org/licenses/by/4.0/). 
calorimetric measurement of the primary energy. The atmospheric depth of the shower maximum (a parameter indicating the mass of the primary particle) can be estimated comparing the cherenkov photon densities measured at two different distances from the shower core. These arrays operate only during clear moonless nights, thus their duty cycle is $\sim 10-15 \%$.

The main problem of these experiments is that energy and mass calibrations have to be performed by means of EAS simulations that are based on hadronic interaction models extrapolated from lower energy collider measurements. Recently the results coming from the LHC experiments are filling this gap, nevertheless collider experiments do not cover the forward region that is the relevant one to describe the EAS development.

In spite of all the mentioned calibration problems the results of last generation experiments allowed an understanding of details of the knee of the primary cosmic ray spectrum. The scenario resulting from such measurements favors an astrophysical interpretation of the knee, even if the resulting picture is not complete and, to further improve our knowledge, high precision experiments are needed. I will show that recent results can, at least qualitatively, be described by elemental spectra with change of slope at energies scaling with Z, even if the recent ARGO-YBJ results [1] cannot be easily included in a simple framework and an additional galactic component dominating the spectrum above $10^{16} \mathrm{eV}$ must be introduced [2,3].

\section{Energy and mass calibration of indirect experiments}

\subsection{Energy calibration}

Surface arrays derive the energy of the primary particle either from the measured value of the number of charged particles $\left(N_{c h}\right)$ either from the measured value of the number of muons $\left(N_{\mu}\right)$ or from a combination of both.

These shower parameters depend not only on the energy of the primary cosmic ray but also on the atmospheric depth crossed by the EAS, thus the zenith angle $\theta$ has to be taken into account. The shower evolution in atmosphere can be treated in two different ways: a first one is based on the experimental data supposing that the arrival directions of cosmic rays are isotropically distributed, the so called "constant intensity cut" [4], while a second one describes the EAS atmospheric evolution by means of a complete simulation.

The main sources of systematic errors in the energy calibration of any indirect experiment are related to the use of a complete EAS simulation and are therefore connected to the two main hypotheses that must be done when running these codes: the mass of the primary particle generating the shower and the hadronic interaction model describing high energy interactions.

Considering the choice of the mass of the primary particle three different strategies are possible:

- the primary energy is calculated for the two extreme values of the primary mass (Hydrogen and Iron). The spectra that are obtained for these two cases represent the upper and lower limits bracketing the "true" spectrum.

- Starting from the primary chemical composition measured at lower energy by direct experiments and assuming its evolution with energy the mean value of the primary mass is calculated as a function of the primary energy. This mean value of the primary mass is then used to convert the experimental observable to primary energy.

- Combining the $N_{c h}$ and $N_{\mu}$ values measured for each event a parameter correlated with the primary mass is evaluated. Then this parameter is used in the conversion from the 


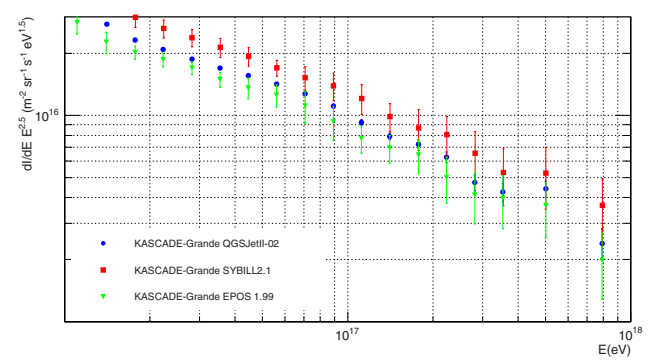

Figure 1. KASCADE-Grande measurements of the all particle energy spectra calibrated by mean of three different hadronic interaction models: QGSJetII-02, Sibyll2.1 and EPOS1.99.

experimental observable to the primary energy [5]. In this way no assumption is made about the primary chemical composition, but a further dependence on the hadronic interaction model is added (in the definition of the parameter correlated with the primary mass).

Cherenkov experiments derive the primary energy from the photon density measured at a fixed distance from the shower core, $200 \mathrm{~m}$ in the case of the TUNKA-133 experiment [6]. Considering TUNKA-133 as reference experiment the correlation between $Q(200)$ and the primary energy is given by: $E_{0}=C Q(200)^{g}$. The parameter $g$ is evaluated by mean of the CORSIKA [7] simulation code, $g=0.94 \pm 0.01$. The value of the constant $C$ is obtained normalizing the integral energy spectrum of each single night of data taking to a reference one measured by the QUEST experiment [8].

The second main source of systematic error in the energy calibration of indirect experiments is the choice of the hadronic interaction model used in the EAS simulation. The center of mass energy of the interaction between an high energy cosmic ray and air nuclei is well above the ones studied at collider experiments. The more often used hadronic interaction models used by current EAS experiments are: QGSJetII-02 [9], Sibyll2.1 [10] and EPOS1.99 [11]. The KASCADE-Grande experiment has evaluated the differences in the energy assignment due to the hadronic interaction model choice [12], finding that they are smaller than $20 \%$. The all particle spectra derived from the KASCADE-Grande data calibrated by means of these three different hadronic interaction models are shown in Fig. 1.

\subsection{Mass calibration}

Due to the intrinsic fluctuations in the EAS development indirect experiments cannot access the mass of every single event, even at shower maximum (where the fluctuations are minimized) the differences between EAS generated by primaries of similar mass (i.e. $\mathrm{H}$ and $\mathrm{He}, \mathrm{He}$ and $\mathrm{CNO}, \mathrm{Si}$ and $\mathrm{Fe}$ ) are smaller than one standard deviation. Studies of the primary chemical composition can be performed using statistical methods like, for instance, the unfolding analysis introduced by the KASCADE experiment [13], or separating events in two mass groups, i.e. light and heavy primaries.

The shower observables sizable to such purpose are:

- the measurement of the shower maximum development. With nowadays techniques this information can be directly obtained only by fluorescence light telescopes, while cherenkov detectors can infer it from the ratio of the photon densities at two different distances from the shower core. 


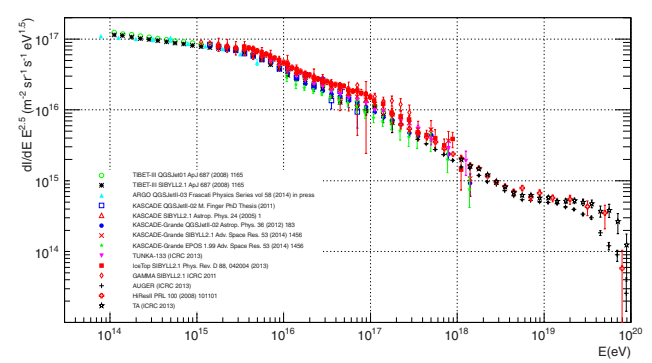

Figure 2. Compilation of all particle energy spectra measurements [1, 5, 6, 12-20].

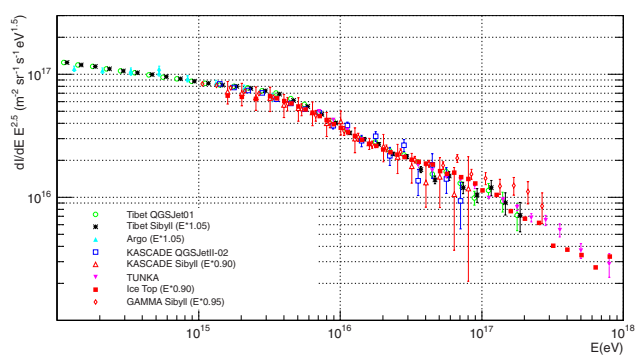

Figure 3. All particle energy spectrum measured in the $10^{14}-10^{18} \mathrm{eV}$ energy range. The energy calibration of the experiments are shifted by an arbitrary amount smaller than the calibration systematic errors. References can be found in the caption of Fig. 2, the energy shifts applied are reported in the legend.

- The ratio between observables representing the charged particle and the muon numbers at observation level.

- A correlation between a parameter sensible to the number of particles at observation level and another one reflecting the shape of the particle lateral distribution.

In order to assign a value of the primary mass to any of the previously indicated experimental observables a complete EAS simulation is needed, therefore all these analyses depend on the high energy hadronic interaction model used.

\section{Experimental results}

Having discussed the main sources of systematic errors affecting the energy and mass measurements performed by indirect experiments, in this section I will discuss some of the more recent results obtained in the $10^{14}-10^{18} \mathrm{eV}$ energy range.

\subsection{All particle energy spectrum measurements}

Figure 2 shows a compilation of the all particle energy spectrum measured by different experiments using different measurement techniques, operating at different height above see level and calibrated by different hadronic interaction models. Looking at this plot we can have the impression of a confused situation, with measurements showing big differences between each other, but simply shifting, as already proposed in [3], the single experiment energies by an amount lower than the previously discussed systematic errors (i.e. $\Delta E / E \leq 20 \%$ ) all the results agree much better, as can seen in Fig. 3. The differences shown in Fig. 2 can thus be 


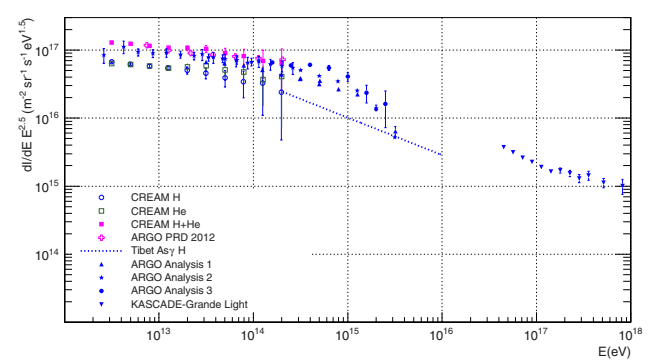

Figure 4. Spectra of the light primaries measured by ARGO-YBJ (the different analysis shown by the collaboration are described in [1]) and KASCADE-Grande. Lower energy direct measurements of the CREAM experiment are shown as a reference. The former measurement obtained by the KASCADE collaboration is not shown in this plot as it was displayed in a non energy calibrated way [21].

attributed to the energy calibration of the experiments and there is a general agreement about the structure of the spectrum showing, in addition to the well known feature known as the knee, two faint but significant structures at $\sim 10^{16} \mathrm{eV}$ and at $\sim 10^{17} \mathrm{eV}$.

\subsection{Mass groups energy spectra}

Indirect experiments can measure the spectra of mass groups of primary cosmic rays by two different approaches that will be described in the following.

A first analysis technique separates the events in two samples according to the measured ratio between the muon and charged particles numbers, converted, by means of the constant intensity cut, to a reference zenith angle. The events sample with a high value of this parameter (called Y by the KASCADE collaboration who introduced this analysis technique [21]) is generated by heavy primaries and the one with low Y values by the light ones. The KASCADE-Grande experiment has then modified this analysis [22, 23] simply calculating the ratio between muon and charged particles numbers in intervals of zenith angles (i.e. atmospheric depth).

A similar approach has been used by the ARGO-YBJ collaboration [1] that, being a full coverage detector operating at higher altitude (i.e. almost near to shower maximum) and not having a muon detector, selected the events on the basis of the ratio between the number of particles measured within a distance of $8 \mathrm{~m}$ from the shower core $\left(N p_{8}\right)$ and the slope $\left(s^{\prime}\right)$ of the lateral distribution function.

In both techniques a selection level must be defined to divide the two samples, this cut has to be defined according to the results of a complete EAS simulation. In the case of the KASCADE and KASCADE-Grande experiments it was shown that the level used to define the two samples affects only the absolute fluxes of the spectra, while eventual spectral features are visible independently from this choice, only enhancing them. For a specified hadronic interaction model the choice of the cut value corresponds to a primary mass, while for a specified primary mass its choice means selecting simulations performed with a particular hadronic interaction model. Thus a spectral feature measured independently from the cut value does not depend on the hadronic interaction model used in the EAS simulation.

Figure 4 shows a summary of the, energy calibrated, KASCADE-Grande and ARGO-YBJ measurements of the light primaries spectra. In the plot also the direct measurements of the $\mathrm{H}$ and $\mathrm{He}$ spectra performed by the CREAM collaboration [24] are shown, their agreement with the low energies measurement of the ARGO-YBJ experiments confirms the validity of 
the energy calibration of this experiment and suggests also the good reliability (at least around $10^{13} \mathrm{eV}$ ) of the hadronic interaction models included in the CORSIKA code.

The KASCADE experiment published [21] the spectra of the "electron rich" (i.e. light primaries) and the "electron poor" (i.e. heavy primaries) events. These spectra are not calibrated in energy (they are shown as a function of the muon density at fixed core distance), but using the integral flux above the change of slope we can identify this feature with the knee of the all particle energy spectrum.

The ARGO-YBJ collaboration has shown [1] the results of three different analysis that differs between each other by an amount lower than the declared systematic errors, all the three spectra show a break well below $10^{15} \mathrm{eV}$.

The KASCADE-Grande experiment published the spectra of the light and heavy components in two slightly different energy ranges with selection cuts defined in order to enhance the spectral features of these two components:

- an hardening of the light component spectrum [23]; at $10^{17.08 \pm 0.08} \mathrm{eV}$ the spectral slope changes from $\gamma=-3.25 \pm 0.05$ to $\gamma=-2.79 \pm 0.08$.

- A steepening of the heavy primaries spectrum [22]; at $\log (E / \mathrm{eV})=16.92 \pm 0.04$ the spectral slope $\gamma$ changes from $\gamma=-2.76 \pm 0.02$ to $\gamma=-3.24 \pm 0.05$.

\section{Discussion}

In this section I present the results of a calculation, based on the work of T. Gaisser et al. [3], of elemental spectra following a Peter's cycle (i.e. with knee energies at constant rigidity). My goal is neither reaching a detailed interpretation of the data nor making any fit to obtain the elemental spectra describing them, I will only search for a qualitative agreement between the data reported in Sect. 3 and the calculated spectra.

The elemental spectra are calculated with an expression [25] describing a power law with index changing from $\gamma_{1}$ to $\gamma_{2}$ at energy $E_{k n e e}$.

$$
\Phi(E)=K E^{\gamma_{1}}\left[1+\left(\frac{E}{E_{\text {knee }}}\right)^{\epsilon}\right]^{\frac{\left(\gamma_{2}-\gamma_{1}\right)}{\epsilon}} .
$$

The parameter $\epsilon$ represent the sharpness of the change of slope, and in these calculations has been kept constant $(\epsilon=10)$ corresponding to a smooth knee.

The elemental fluxes have been calculated with the following hypotheses:

1) the absolute normalization is given by the intensities measured by CREAM [24] at $10^{13} \mathrm{eV}$.

2) The slopes of the $\mathrm{H}$ and $\mathrm{He}$ spectra before the knee are the ones measured by CREAM, the slope of the He spectrum is used also for heavier elements.

3) All the spectra suffer the same change of slope, values from $\Delta \gamma=0.5$ to $\Delta \gamma=0.7$ have been used.

4) The knee energy has been calculated following a Peter's cycle: $E_{\text {knee }}(Z)=Z \times$ $E_{\text {knee }}(H)$. The Hydrogen knee is fixed at two different energies: $E_{\text {knee }}(H)=6.5 \times$ $10^{14} \mathrm{eV}$ following the ARGO-YBJ results and $E_{\text {knee }}(H)=1.5 \times 10^{15} \mathrm{eV}$ attributing the knee of the all particle spectrum to a light element, i.e. either $\mathrm{H}$ or $\mathrm{He}$.

5) To account for the hardening of the light elements spectrum a flatter $\mathrm{H}$ component ( $\gamma=-2.66$ ) becoming dominant at $\sim 10^{17} \mathrm{eV}$ is added. 


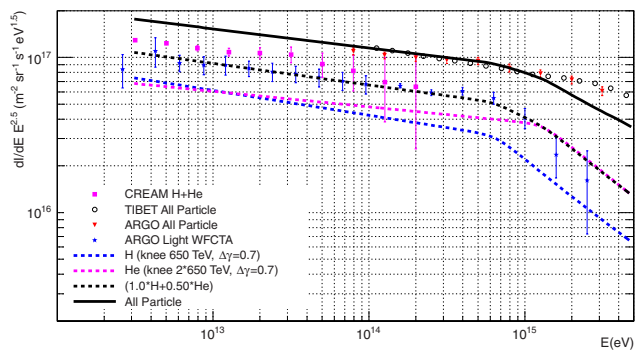

Figure 5. All particle and light elements spectra measured by the ARGO-YBJ experiment [1] compared with the calculations described in Sect. 4.

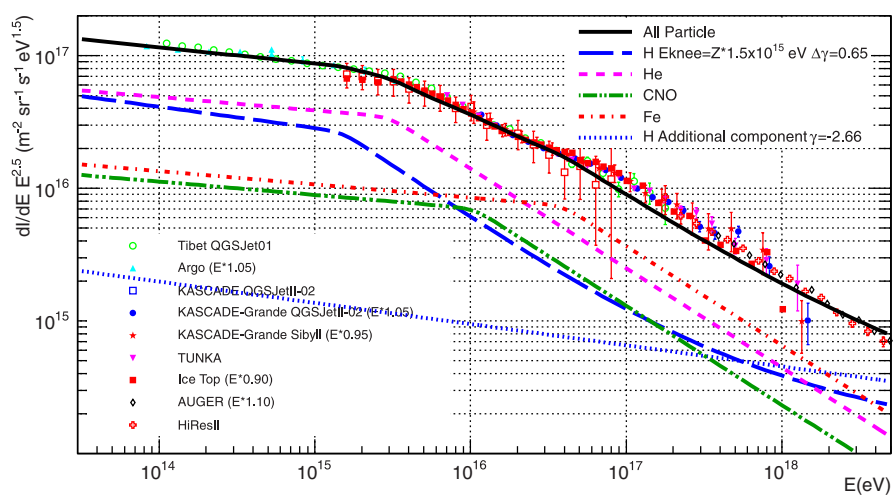

Figure 6. Summary of all particle spectrum measurements, shifted by an arbitrary amount (reported in the legend), and compared with the calculations described in Sect. 4.

Comparing the results of these calculations with the mass group spectra we must always keep in mind that the intensities measured with these algorithms aim to enhance spectral feature and not to determine the absolute fluxes.

In Fig. 5 the ARGO-YBJ light and all particle spectra [1] are shown together with the results of the previously described calculations. We can see as the sharp knee of the light elements spectrum can be described fixing a huge value for the change of slope $(\Delta \gamma=0.7)$, but summing all elements to calculate the all particle spectrum we cannot describe the TibetIII [15] and ARGO-YBJ measurements. This disagreement is smaller assuming that the energy of the knee scales with the mass of the primaries (A) or if we use harder spectra for elements heavier than $\mathrm{He}$, but it is in any case difficult to have a good description of both the light and the all particle spectra. If the ARGO-YBJ results will be confirmed an additional component must be invoked.

Extending the elemental spectra calculations from $10^{14} \mathrm{eV}$ to $5 \times 10^{18} \mathrm{eV}$ we can compare the expectations with the measurements in a wide energy range, in Fig. 6 I show the calculated fluxes and the experimental data shifted by an arbitrary scale. The shown fluxes correspond to: $\mathrm{H}$ (blue long-dash line), He (magenta short-dash line), C (green dashdotted line), Fe (red dotted line) and their sum (black thick solid line). We can see that the knee structure is well reproduced by elemental spectra following Peter's cycle, but further details must be clarified by future experiments: 1) which element is dominating the spectrum 
at the knee? 2) If all the claimed results are confirmed, is there a contradiction between measurements performed at high altitudes and at sea level? 3) Which is the exact energy of the knee? 4) The knees of different elements scale with the charge (Z) or with the mass (A) of the primaries? 5) At energies smaller than the knee are the spectral indexes of all elements (except $\mathrm{H}$ ) equal or are they different? 6) Is the change of slope at the knee the same for all elements?

Thus new measurements are required, these can be performed either by direct, high acceptance measurements or by indirect high resolutions arrays. Both techniques have their advantages and limitations:

- direct measurements. Long duration satellite experiments, like the foreseen iss-cream [26], will be able to collect enough statistics to cover the knee energy and will have the charge resolution to separate all elements. Their main limitation will be the energy resolution $(\sim \Delta E / E \sim 40 \%)$ as they will have strong constrain on their mass, therefore calorimetric detectors will contain only a small fraction of the shower generated the primary particle interaction. Furthermore no man made sources will be available at these energies, thus the absolute energy calibration will be performed indirectly by simulations.

- Indirect measurements. The main limitation of EAS experiments will always be the EAS development fluctuations. The best way to minimize them is to locate the array at high altitudes detecting showers at their maximum development. This is the goal of the LHAASO experiment [27] that will be constructed in China, in the Sichuan province at $\sim 4300 \mathrm{~m}$ a.s.l.. This experiment will sample the electromagnetic and muonic shower components with an unprecedented active surface reaching therefore a huge resolution.

\section{References}

[1] G. Di Sciascio on behalf of the ARGO collaboration, Frascati Physics Series 58 (2014) in press

[2] A.M. Hillas, J. Phys. G: Nucl. Part. Phys. 31, R95 (2005)

[3] T.K. Gaisser et al., Front. Phys. 8(6), 748 (2013)

[4] J. Hersil et al., Phys. Rev. Lett. 6, 22 (1961); D.M. Edge et al., J. Phys. A 6, 1612 (1973)

[5] W.D. Apel et al., Astropart. Phys. 36, 183 (2012)

[6] V.V. Prosin et al., Nucl. Instr. Meth. A 756, 94 (2014)

[7] D. Heck et al., Report FZKA 6019, Forschungszentrum Karlsruhe (1998)

[8] E.E. Korosteleva et al., Nucl. Phys. B (Proc. Suppl.) 165, 6837 (2007)

[9] S. Ostapchenko, Phys. Rev. D 74, 014026 (2006)

[10] E.-J. Ahn et al., Phys. Rev. D 80, 094003 (2009)

[11] K. Werner, F.M. Liu, T. Pierog, Phys. Rev. C 74, 044902 (2006)

[12] W.D. Apel et al., Advances in Space Research 53, 1456 (2014)

[13] T. Antoni et al., Astropart. Phys. 24, 1 (2005)

[14] M.G. Aartsen et al., Phys. Rev. D 88, 042004 (2013)

[15] M. Amenomori et al., ApJ 678, 1165 (2008)

[16] M. Finger, PhD Thesis, Karlsruhe University (2011)

[17] A.P. Garkaya et al., J. Phys. G: Nucl. Part. Phys. 35, 115201 (2008)

[18] A. Aab et al., arXiv:13075059v1 (2013)

[19] R.U. Abbasi et al., Phys. Rev. Lett. 100, 101101 (2008)

[20] T. Abu-Zayyad et al., arXiv:1305:7273 (2013) 
[21] T. Antoni et al., Astropart. Phys. 16, 373 (2002)

[22] W.D. Apel et al., Phys. Rev. Lett. 107, 171104 (2011)

[23] W.D. Apel et al., Phys. Rev. D 87, 081101(R) (2013)

[24] Y.S. Yoon et al., ApJ 728, 122 (2011)

[25] S.V. Ter-Antonyan and L. Haroyan, arXiv:hep-ex/0003006 (2000)

[26] E.S. Seo et al., Proc. of the 33rd ICRC (Rio de Janeiro, Brasil), \#0629 (2013)

[27] Cao Z., Chinese Physics C 34, 249 (2010) 